\title{
Mechanical Analysis of Stiffened Plates Made by Layered Braided Composite
}

\author{
Zi-Hao WANG ${ }^{1, *}$, Jian-Guo WU ${ }^{2}$ and Long WANG ${ }^{2}$ \\ ${ }^{1}$ School of Aeronautic Science and Engineering, Beihang University, Beijing 100191, China \\ ${ }^{2}$ Science and Technology on Reliability and Environmental Engineering Laboratory, Beijing Institute \\ of Structure and Environment Engineering, Beijing 100076, China
}

*Tel: 18810661150

Keywords: layered braided composite, FEM, stiffened plates model, mechanical properties.

\begin{abstract}
Layered braided composite is widely used in structures. The mechanical analysis of this material is more complicated than that of laminated plate composite materials, so the accurate solution of structure made of this material is fairly difficult to obtain. In this paper, a simplified FEM model is used to analyze the mechanical properties of the structure composed by braided composite. A sole cell model in microscopic scale is established to obtain the basic material properties of braided composite. Furthermore, a simplified stiffened plate model is established to reduce the computational work. Finally, the effect of different design parameters on the mechanical properties of stiffened plates is also analyzed.
\end{abstract}

\section{Introduction}

Composite materials, especially new composite materials are increasingly widely used, even in the main load-bearing structure. As a result, the mechanical research of composite material structure becomes very important $[1,2]$.

Compared with traditional composite materials, braided composites have better mechanical properties, especially in anti-shock and anti-high temperature [1, 3, 4].

A stiffened plate model shown in Figure 1 is taken to be analyzed. The plate in this model consists of braided composite laminate. The research aims at discussing the effect of different design parameters, including separate distance, layer amounts, the effect of insufficient deposition and ply orientation angle.

\section{Method}

As most researchers used, finite element method (FEM) by ANSYS software is used to achieve the research goal [5].

The scale of basic cell of braided composite plate is fairly tiny, thus the structure will contain innumerable basic cells. It is impractical to carry out FEM analysis of the structure in the level of basic cell. Thus, the analysis is made by two steps. Firstly an accurate microscale cell model is built to get the basic material properties, the material characteristics obtained in step 1 is shown in table 1; accordingly, a simplified structure model is established to make the mechanical analysis. 


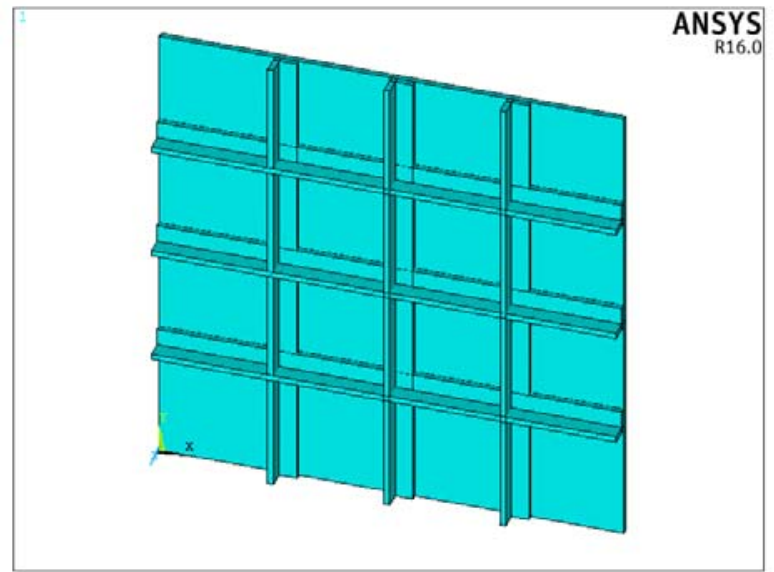

Figure 1 Sketch of Stiffened Plate Model

\section{Microscale Cell Model}

On the basis of the real form of braided composite observed under microscope, the basic cell model is built. Cell model contains both braided fiber and deposition substrate. In consideration of structural symmetry, the FEM model is simplified into a quarter of the original model with symmetric boundary condition. Thus the minimum cell model is obtained. This process is showed in Fig. 2.

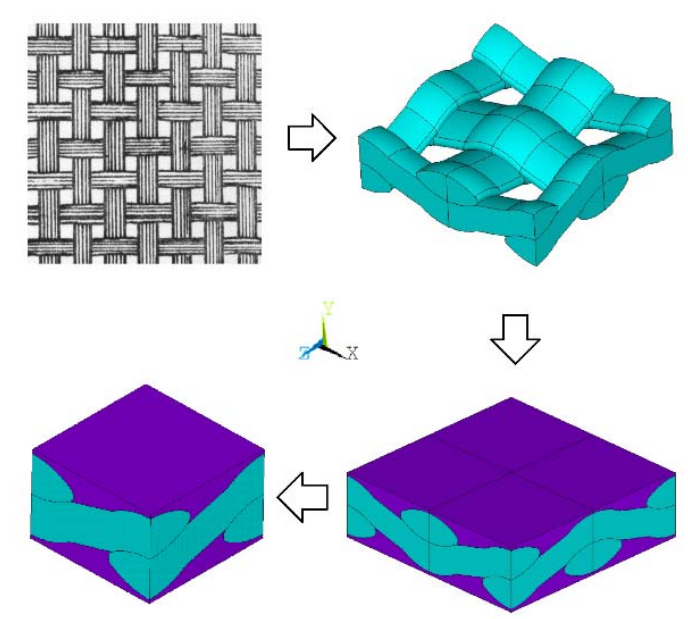

Figure 2 Cell Model on the Basis of Real Structure

Unit loads are defined along the z-axis while symmetric boundary conditions are adopted on the $\mathrm{x}-\mathrm{y}$ and $\mathrm{y}-\mathrm{z}$ surfaces. Macroscale material properties can be obtained based on the displacement along three axes.

By this method, the material properties of the laminate can be obtained from the material properties of fiber and substrate.

The material property of fiber refers to T700 carbon fiber, for which Young's modulus $E_{f}=230 \mathrm{GPa}$, and for substrate Es=1GPa. 
Thus the material properties are obtained by the result of the cell model as follows:

Table 1 Material Properties (Unit: [GPa])

\begin{tabular}{l|l|l|l|l|l}
\hline EX & Ey & Ez & GXZ & GYZ & GXY \\
\hline 107.06 & 18.80 & 107.06 & 56.48 & 31.60 & 31.60 \\
\hline
\end{tabular}

\section{Macroscale Laminate Model}

As previously described, a detailed structure contains innumerable basic cells. It is impractical to carry out FEM analysis of the structure in the level of basic cell. In order to save the calculating time, a simplified model is established in the following for analysis.

The material is considered as orthotropic and homogeneous when building the FEM model. Thus a simplified model of laminate transforming from braided composite structure can be made.

ANSYS is used to carry out the FEM process, the element Solid185 with Key Option $3=1$ is taken, which is a layered solid mode. This mode is appropriate for layered style solid structure such as laminate. The section parameters such as layer amounts and ply orientation angle can be set with SECXx series commands.

A parametric model is built to find the effect of different design parameters. The model can be easily redefined with different parameter settings. A standard model is built as a contrast at the beginning with parameters as shown in table 2.

Table 2 parameters of the standard model

\begin{tabular}{l|l}
\hline PS (Stiffened plate size)/ [mm] & 5000 \\
\hline SD (Separate distance)/ [mm] & 2500 \\
\hline FT (Plate thickness)/ [mm] & 40 \\
\hline Layer amounts & 20 \\
\hline Single layer thickness/[mm] & 2 \\
\hline Deposition condition & $100 \%$ \\
\hline Ply orientation angle & $0 / 90$ \\
\hline
\end{tabular}

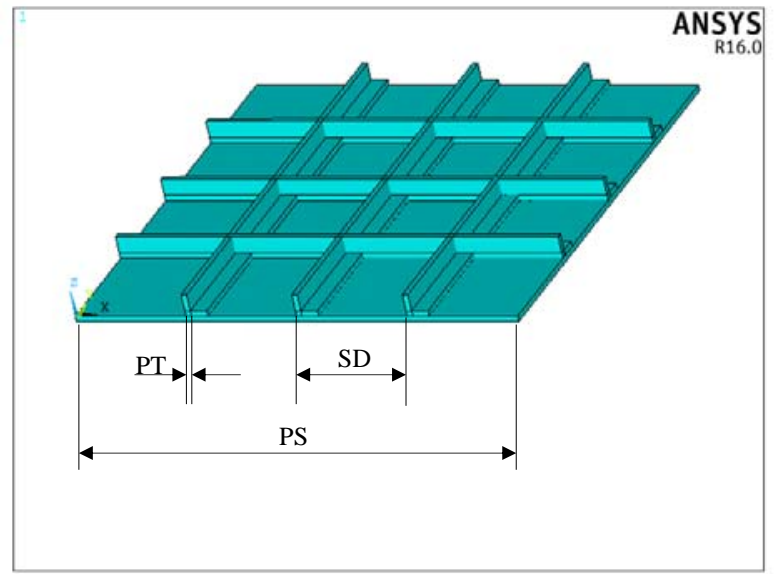

Figure 3 Geometrical Feature Parameters 
The geometry form and some insignificant parameters remain invariable during the following analysis. The aim of the research is to determine the effect of the parameters about stiffened plates, including separate distance, layer amounts, deposition degree and the ply orientation angle.

The standard model includes a basal plate with XY weaving direction, with the rivets hammered into it along Z-axis direction. The perpendicular stiffened plate has XZ and YZ weaving direction respectively. It is noticeable that only 2 stiffened plates in $\mathrm{X}$ and $\mathrm{Y}$ directions are considered to simplify the calculation without loss of generality.

The model is clamped at four edges. Unit pressure loads (1.0 Pa) are applied on the bottom surface.

\section{Results}

\section{Results from Standard Model}

The solution of the above model under unit loads previously described is obtained. The maximum Von Mises stress is located at the top of the stiffened plate, the value of which is $1648.74 \mathrm{~Pa}$. If the parts of rib plates are concealed, the maximum stress at basal plate is only about 750Pa. This means the rib plate bears the major part of the load and protects the basal plate.

\section{The Effect of Separate Distance}

The effect of separate distance is discussed in this section, with the size of stiffened plate, plate thickness and layer amounts remaining constant. The distance between the two rib plates in the structure is changed from $2000 \mathrm{~mm}$ to $3000 \mathrm{~mm}$ with the step of $200 \mathrm{~mm}$, and the results of Von Mises stress are obtained from these models along with the standard model described in chapter 3.1.

The relation between the maximum Von Mises stress and separate distance is shown in Fig. 4.

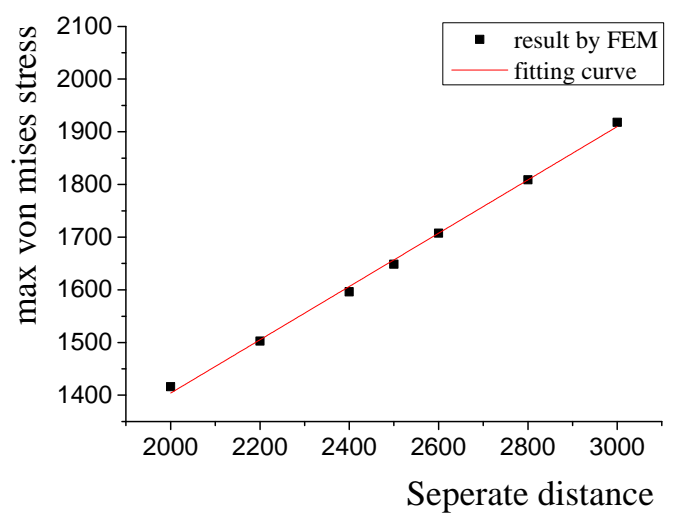

Figure 4 Maximum Von Mises Stress versus Separate Distance

The result shows that the maximum Von Mises stress and separate distance approximately follow linear relation as shown in Eq. (1), and the change of separate distance will not affect the stress distribution:

$$
y=0.5059 d+392.1
$$

In Eq. (1), $d$ represents the value of separate distance and $y$ represents the maximum Von Mises 
stress.

\section{The Effect of Layer Number}

The effect of layer number is analyzed in this section. Four conditions are taken into account in the study which have $15,20,25,30,35$ layers respectively, the thickness of plate is $30,40,50,60$, 70mm correspondingly.

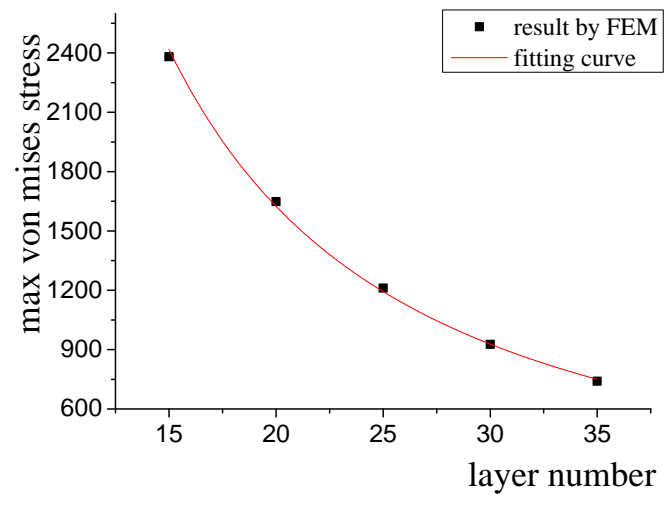

Figure 5 Maximum Von Mises Stress versus Layer Number

The relation between the maximum Von Mises stress and layer number is shown in Fig. 5. The relation can be fitted into a power function as shown in Eq. (2), in which $m$ represents layer number:

$$
y=102022 m^{-1.382} .
$$

The maximum Von Mises stress changes remarkably with the change of layer number. Because the thickness of single layer remains constant, the total thickness of plate changes accordingly. With the increase of the layer number, the slope of line in Fig. 5 decreases. When the layer number is large enough, the increase of layer number will not improve strength of the structure further, while it is noteworthy that the structural weight increases linearly accordingly. So it might be advisable to strengthen the structure by add layers only when the number of layers is not too large.

\section{The Effect of Insufficient Deposition}

In the manufacturing process, the layered braided composite material is possible to be deposited insufficiently. The effect of this kind of defect is discussed in this section. 


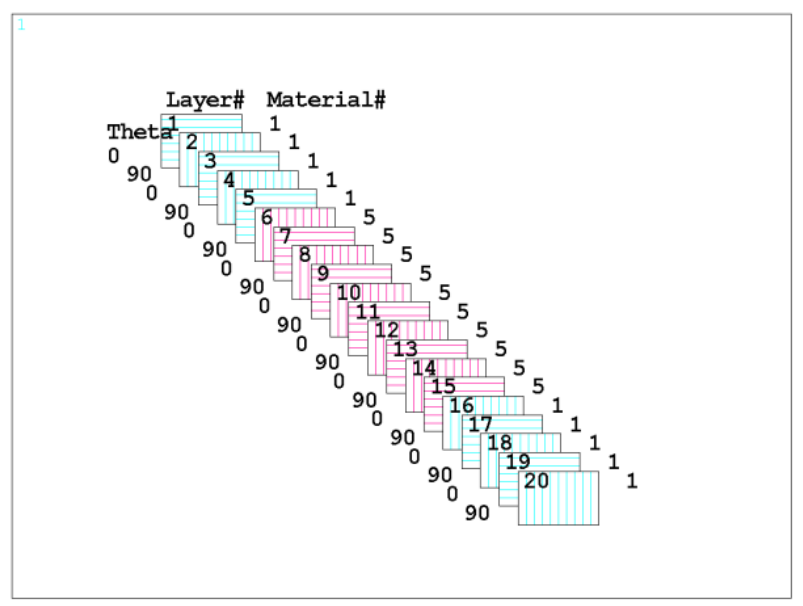

Figure 6 Sketch of Deposition Status

As an example shown in Fig. 6, the structure is deposited insufficiently in the middle of the plate (red marked layers). The Young's modulus of these layers is assumed to decrease to 50 percent of that of completely deposited status shown in Table 1.

Four cases of structures, in which 6, 8, 10 and 12 layers are deposited insufficiently in the plate, are studied. The results are shown in Fig. 7.

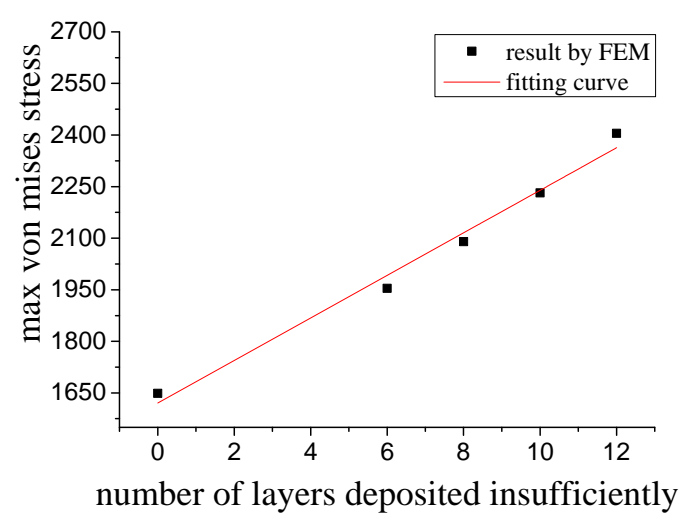

Figure 7 Maximum Von Mises Stress versus Number of Insufficiently Deposited Layers

It can be concluded that the structural strength is reducing with the increase of proportion of insufficiently deposited layers. The relation between the maximum Von Mises Stress and number of insufficiently deposited layers can be fitted into a linear function as shown in Eq. (3), in which $n$ represents the number of layers deposited insufficiently:

$$
y=61.89 n+1620.4
$$

\section{The Effect of Ply Orientation Angles}

The effect of ply orientation angles is discussed in this section. Two styles of structures with ply angles of 0,45 and $0,30,60$ degrees are taken into consideration respectively. The results are 
compared with the standard model with 0 and 90 degrees of ply orientation angles. The results of analysis of the three kinds of structures are shown in Fig. 8.

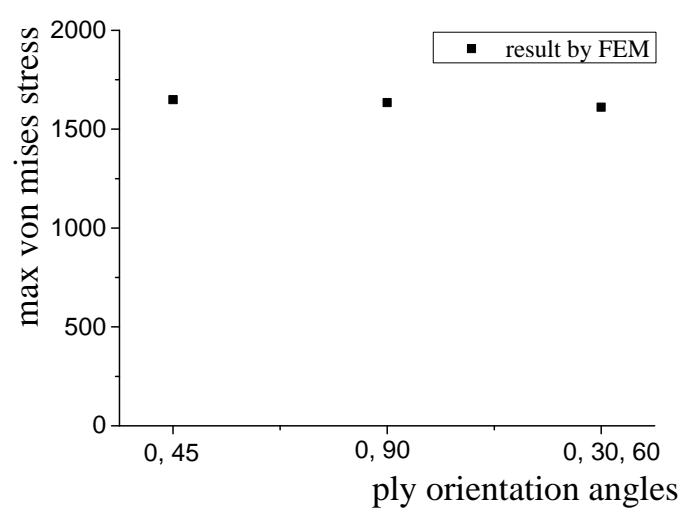

Figure 8 Maximum Von Mises Stress versus Different Ply Orientation Angles

From Fig. 8, it can be concluded that the effect of ply orientation angles is very tiny. The vibration range of maximum Von Mises stresses among different structures is within 2\%. Because the change of ply angles will increase the degree of manufacturing difficulty, it is not worthy to change the ply orientation angles in practice.

\section{Conclusion}

The effect of four kinds of design parameters of stiffened plate is studied by finite element method. The effect of different parameters on the strength of structure is different. From the analysis above, the relationship between strength of the structure and the parameters considered in this study can be determined in the following.

The effect of ply orientation angles is very tiny. It is not necessary to change the ply orientation angle in manufacturing process.

The effects of separate distance, layer number and insufficient deposition are relatively obvious. The relations between these design parameters and the maximum stresses of the structure can be expressed in Eq. (1)-(3). Within the scope of design parameters in the analysis, the effect of layer number is most remarkable. As shown in Fig. 5, the maximum stress varies from 740 to $2380 \mathrm{~Pa}$.

For the design parameters considered in this paper, the change of the parameters will lead to the variation of weight and the cost of manufacturing. It is necessary to consider the strength, weight and the cost of manufacturing when optimizing the design of the structure.

\section{Reference}

[1]. Brigante D. New CompositeMaterials[J]. Nature, 2014, 442(7100):282-286.

[2]. Thostenson E T, Ren Z, Chou T W. Advances in the science and technology of carbon nanotubes and their composites: a review[J]. Composites Science \& Technology, 2001, 61(13):1899-1912.

[3]. Dandekar C R, Shin Y C. Modeling of machining of composite materials: A review[J]. International Journal of Machine Tools \& Manufacture, 2012, 57(2):102-121.

[4]. LU Zixing, YANG Zhenyu,LI Zhongping. Development of investigation into mechanical 
behaviorof three dimensional braided composites[J]. ActaMateriaeCompositaeSinica, 2004, 21(2):1-7.

[5]. LIAO yingqiang, SU jianhe, KE shanliang. Application of Ansys in composite simulation analyses[J]. Fiber glass, 2006(1):63-66. 